\title{
Dynamic Aberration-corrected STEM of Bimetallic Nanocatalysts during Surface Diffusion
}

\author{
Volkan Ortalan ${ }^{1}$, Chang Wan Han $^{1}$, Rodolfo Zanella ${ }^{2}$, Antonio Aguilar-Tapia ${ }^{2}$ \\ 1. School of Materials Engineering, Purdue University, West Lafayette, IN, USA \\ 2. Centro de Ciencias Aplicadas y Desarrollo Technologico, Universidad Nacional Autonoma de \\ Mexico, Mexico City, Mexico
}

Fundamental investigations of catalyst structures and the mechanisms of catalytic reactions requires characterization imaging of surfaces at the atomic scale and probing the structures and energetics of the reacting molecules as they function under reaction condition on varying time and length scales. Highangle annular dark field (HAADF) STEM is an indispensable technique for analyzing heterogeneous catalysts, in particular those comprising high atomic number $(Z)$ metallic nanoparticles (NPs) dispersed on low-Z supports [1]. Readily interpretable atomic-scale Z-contrast imaging with high spatial resolution spectroscopy, including X-ray energy dispersive spectroscopy (EDS) and electron energy loss spectroscopy (EELS), allows researchers to investigate structural information such as dimensions, morphologies, and size distribution of catalytic NPs as well as their material chemistry (e.g., chemical composition, bonding of catalytic particles with supports at interface, etc.).

The formation of extremely small supported metal clusters, starting from the simplest case of essentially molecular, atomically dispersed catalysts -in our case, supported gold, iridium, rhodium complexes, has been recorded by high-resolution STEM imaging at the atomic scale in real time. The bond breaking and forming that catalytic clusters readily undergo is referred to as fluxionality (changes in local morphology of the catalyst) and occurs typically on time scales ranging from picoseconds to seconds depending mostly on the size, interaction with the support, temperature and environment [2]. Investigations of this dynamic nature of catalytic structures are extremely significant to develop a fundamental understanding of activity-structure relationship. This dynamic nature of catalysts clusters is illustrated for an 8-atom Ir clusters in Figure 1.

Metal nanoparticles with precisely controlled size and composition are highly attractive for heterogeneous catalysis. However, a long-standing problem is the tendency of nanoscale particles of such elements to coalesce ('sinter') into much larger aggregates under process conditions [3]. This leads to deactivation of the catalyst caused by the reduction of the surface area of the precious metal available to the reactant species. Despite the huge losses to industry and to the environment caused by sintering, a detailed atomic-level picture is not yet available. Therefore, detailed structural-dynamic information achieved from a direct and real-time in situ observation of sintering could provide the basis for fully elucidating the sintering mechanism [4] (Ostwald ripening and particle migration and coalescence) in supported metal catalysts and, as a result, controlling them for catalytic application. The STEM Zcontrast [5] images in the figure 2b-c are chemically sensitive allowing us to probe 3D morphology and chemistry of the nanoparticles with single atomic sensitivity. Figure 2d1-4 illustrates atomic scale images of sintering process starting from extremely small clusters (a single metal atoms) to larger nanoparticles. To understand the mechanisms of cluster formation from small clusters, HAADF-STEM images were recorded using Cs-corrected STEM. Heating experiments were also performed by using Protochips Co. AduroTM MEMS-based heating technology, which provides highly stable operation at temperature that does not compromise the resolution of the microscope [6]. In this presentation, obtained 
dynamic results for the fluxionality and surface diffusion of the small size clusters in heterogeneous catalysts at high spatiotemporal resolution will be discussed [7].

\section{References:}

[1] P.D. Nellist PD et al, Science 2004, 305, 1741.

[2] A. Vargas et al, Phys Rev B. 2009, 80, 195421.

[3] R.A Betta Dalla, R.C. McCune, J.W. Sprys, Industrial \& Engineering Chemistry Product Research and Development 1976, 15, 169-172.

[4] R.S. Goeke, A.K. Datye, Topics in Catalysis 2007, 46, 3-9

[5] V. Ortalan, et al, Nature Nanotechnology 2010, 5, 843-847.

[6] L.F. Allard, et al, Microscopy Research and Technique, 72, (2009) 208-215

[7] This work was supported by the Designing Materials to Revolutionize and Engineer our Future

(DMREF) program of the National Science Foundation (CBET-1437219).

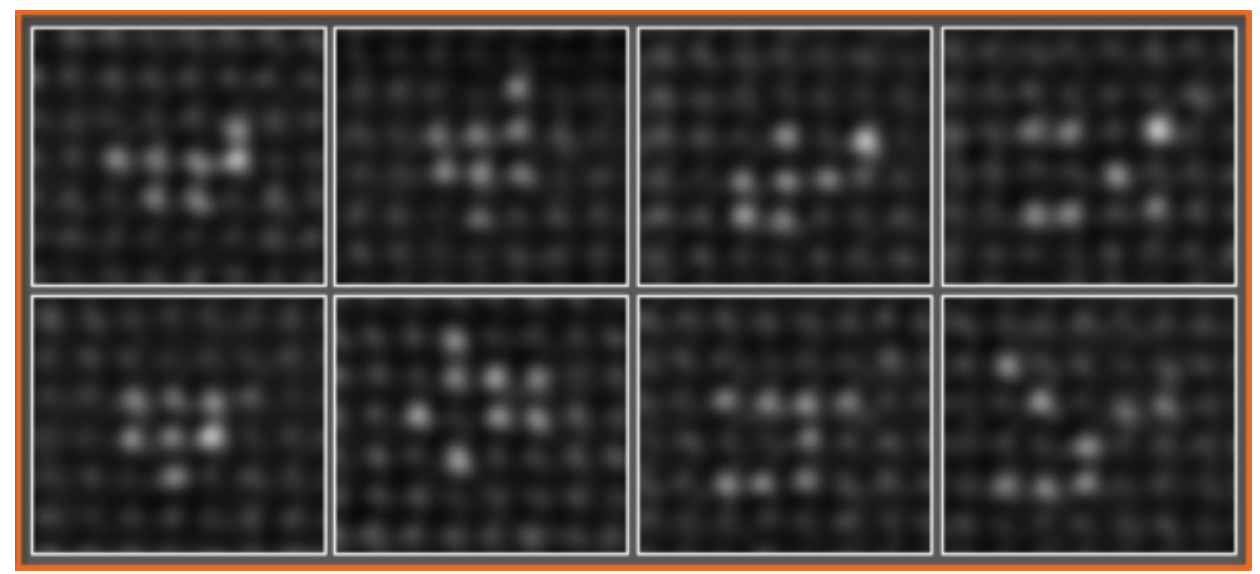

Figure1: Fluxionality of an 8-atom cluster on $\mathrm{MgO}(100)$ surface.

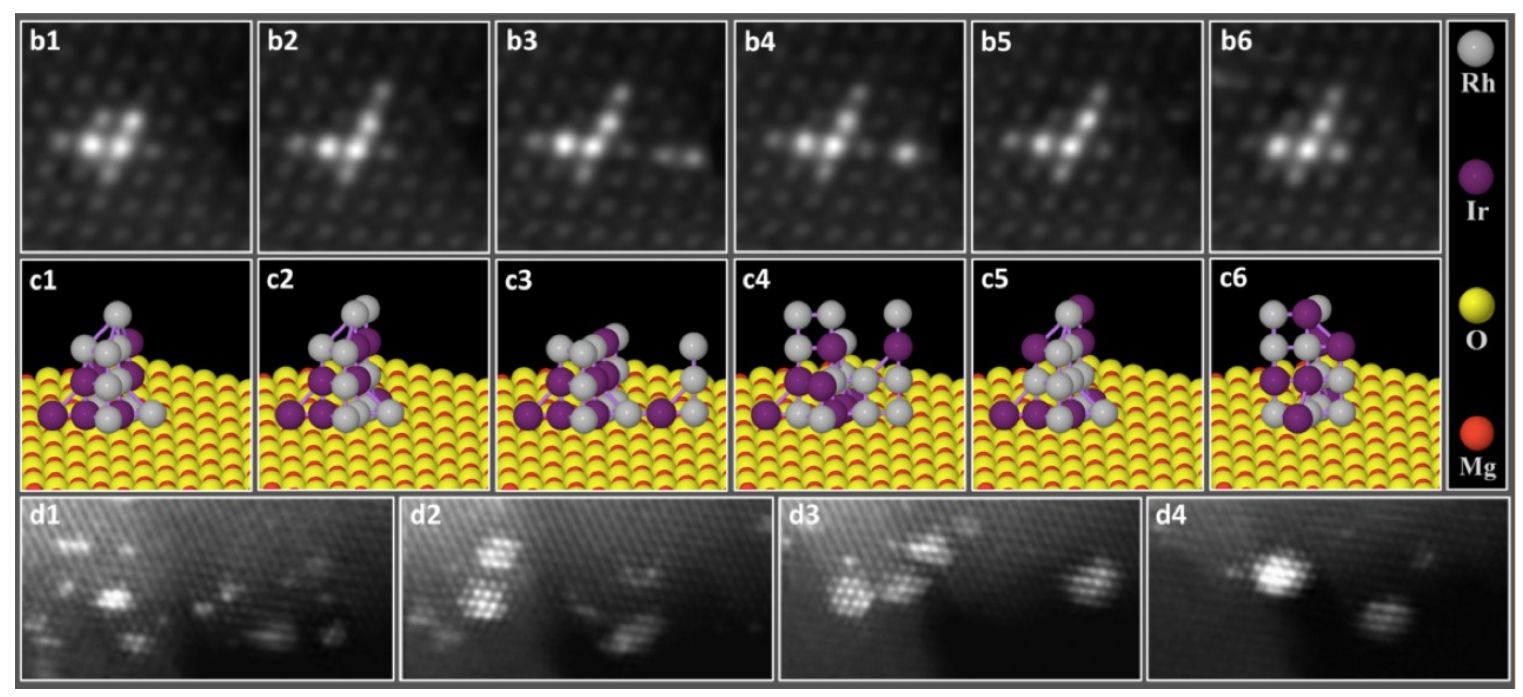

Figure2: bc1-6) Dynamic atomic resolution STEM images of a RhIr nanoparticle and 3D model showing the structures of the nanoparticle. d1-4) Dynamic investigation of sintering process of $\mathrm{MgO}$ supported Ir catalysts. 